\title{
Review Board Process Date
}

National Cancer Institute

\section{Source}

National Cancer Institute. Review Board Process Date. NCI Thesaurus. Code 693664.

The date (and time) on which the Institutional Review Board (IRB) made a determination concerning an aspect of the study. 